Arq. Bras. Med. Vet. Zootec., v.67, n.3, p.809-818, 2015

\title{
Frequências de cortes em nove leguminosas forrageiras tropicais herbáceas cultivadas ao sol e sob plantação florestal
}

\author{
[Cutting frequencies of nine herbaceous tropical forage legumes cultivated under full sun light \\ and under a forest plantation] \\ M.L.F. Nicodemo ${ }^{1}$, F.H.D. Souza ${ }^{1}$, J.R.M. Pezzopane ${ }^{1}$, J.C.T. Mendes ${ }^{2}$, \\ P. Tholon ${ }^{1}$, P.M. Santos ${ }^{1}$ \\ ${ }^{1}$ Embrapa Pecuária Sudeste - São Carlos, SP \\ ${ }^{2}$ Escola Superior de Agricultura Luiz de Queiroz - USP - Piracicaba, SP
}

\begin{abstract}
RESUMO
O objetivo deste trabalho foi estudar a produção e o valor nutritivo de nove espécies de leguminosas forrageiras herbáceas tropicais, cultivadas sob dois níveis de irradiação luminosa e seis frequências de corte na estação chuvosa, em Anhembi (SP), Brasil. Parcelas puras foram estabelecidas ao sol e sob plantação de Eucalyptus grandis, com 8 anos de idade, com 1.000 árvores/ha, que interceptou mais de $80 \%$ da radiação luminosa incidente. Avaliou-se a taxa de acúmulo de matéria seca, a proteína bruta e a digestibilidade in vitro da matéria seca em amostras de plantas colhidas em intervalos de 30, 60, 90 e 180 dias, com cortes adicionais aos 120 e 150 dias. Sob pleno sol, as leguminosas testadas toleraram cortes mais frequentes (intervalos de 30 - 90 dias), exceto o Lablab. Na sombra, o grau de tolerância variou conforme a espécie; em termos de produção de MS e de persistência, a Centrosema e a Puerária se destacaram dentre as demais leguminosas comparadas, quando os cortes foram realizados em intervalos de 60 dias, enquanto o Arachis e o Estilosantes foram os mais produtivos quando os cortes ocorreram em intervalos de 120 dias, mantendo o valor nutricional. O Alysicarpus e a Aeschynomene não persistiram sob o nível de sombreamento que prevaleceu nesse experimento.
\end{abstract}

Palavras-chave: qualidade, produtividade, sistema silvipastoril

\begin{abstract}
The aim of this study was to evaluate the production and nutritive value of nine tropical legumes under two light intensities and six cutting regimens in the wet season, in Anhembi (SP) - Brazil. Pure stands were established in full light and under an eight-year-old eucalyptus plantation with 1.000 plants per hectare. The trees intercepted over $80 \%$ of the incident radiation. Dry matter herbage accumulation, dry matter in vitro digestibility and crude protein under the cutting intervals 30, 60, 90 and 180 days, with additional cuttings at 120 and 150 days were evaluated. With the exception of Lablab, all other tested legume species tolerated higher cutting frequency under when grown under full sun light then under shade. The degree of shade-tolerance varied according to the species; Centrosema and Pueraria produced well at cutting intervals of 60 days, showing higher dry matter production and persistence while Arachis and Stylosanthes showed better results under longer cutting intervals, keeping the nutritional value, and can be indicated for differential grazing. Alysicarpus and Aeschynomene did not persist under the prevailing conditions.
\end{abstract}

Keywords: nutritional value, productivity, silvopastoral

\section{INTRODUÇÃO}

A área de florestas plantadas está em expansão no Brasil. No Estado de São Paulo, a área ocupada por florestas de eucalipto alcançou mais

Recebido em 8 de agosto de 2013

Aceito em 10 de junho de 2014

E-mail: marialuiza.nicodemo@embrapa.br de um milhão e duzentos mil hectares em 2010 (Associação..., 2011). Bovinos, equinos e ovinos pastejam o sub-bosque, como forma de reduzir o risco de incêndios florestais, pelo controle da biomassa herbácea e como fonte de renda complementar. A baixa disponibilidade de luz no 
sub-bosque, entretanto, compromete a produção de biomassa aérea, reduz a formação de raízes o que aumenta a susceptibilidade ao estresse - e diminui a taxa de perfilhamento em gramíneas (Guenni et al., 2008). Mesmo considerando a baixa produtividade, a grande quantidade de área disponível aumenta o interesse por técnicas que melhorem a disponibilidade de forragem de alta qualidade sob essas condições.

A estratégia de manejo das forrageiras deve ser adaptada às condições de sombra. Plantas sombreadas necessitam de maior tempo de recuperação após a desfolha que plantas a pleno sol. Chin (1998) indicou intervalos entre corte de 8 a 12 semanas para forrageiras sob plantações florestais onde a interceptação de luz alcançava de 90 a $80 \%$ com o fechamento do dossel. Andrade et al. (2001) reportaram o declínio da produtividade de gramínea no sub-bosque de eucalipto associado à baixa disponibilidade de nitrogênio. A introdução de leguminosas no subbosque pode melhorar a ciclagem de nutriente, fornecendo alimento de alto valor nutricional. Estudos indicaram uma gama de leguminosas tropicais com potencial de uso em ambientes sombreados (Congdon e Addison, 2003), mas é necessário selecionar espécies adaptadas ao manejo e às condições edafoclimáticas da região de interesse. As respostas de forrageiras ao corte foram estudadas geralmente a pleno sol, sendo necessários ajustes para seu uso na sombra. Este estudo avaliou a resposta à frequência de corte na estação chuvosa de forrageiras tropicais, ao sol e à sombra, para subsidiar a otimização do seu manejo.

\section{MATERIAL E MÉTODOS}

O experimento foi conduzido na Estação Experimental de Ciências Florestais da EsalqUSP, em Anhembi, SP $\left(22^{\circ} 40^{\prime} \mathrm{S}, 48^{\circ} 10^{\prime} \mathrm{W}, 455 \mathrm{~m}\right.$ a.n.m.), em área de solo Latossolo VermelhoAmarelo Distrófico, de baixa fertilidade, com $18 \%$ de argila, $9 \%$ de silte e $73 \%$ de areia. O clima prevalecente na região é do tipo Cwa (Köppen); a precipitação média anual é de $1.100 \mathrm{~mm}$; a deficiência hídrica anual, de $25 \mathrm{~mm}$; e a temperatura média anual, de $20,9^{\circ} \mathrm{C}$. Foram plantadas nove espécies de leguminosas herbáceas, distribuídas em quatro blocos ao sol e quatro blocos à sombra de Eucalyptus grandis, plantado há oito anos em espaçamento de $3 \mathrm{~m} \mathrm{x}$ $2 \mathrm{~m}$, submetido a desbaste de $40 \%$, resultando em
1.000 plantas/ha. As leguminosas estudadas foram Aeschynomene villosa cv. Vilomix, Alysicarpus vaginalis "comum", Arachis pintoi cv. Mandobi, Calopogonium mucunoides "comum", Centrosema schiedeanum cv. Belalto, Clitoria ternatea "comum", Lablab purpureum cv. Rongai, Estilosantes Campo Grande (Stylosanthes capitata + S. macrocephala, 80:20) e Pueraria phaseoloides "comum". As sementes foram inoculadas com cepas específicas Rhyzobium spp. antes do plantio, realizado em 03/12/2008, com densidade de semeadura que objetivou a obtenção de 40 plântulas por $\mathrm{m}^{2}$. Cada parcela media $2,5 \mathrm{~m}$ x $5 \mathrm{~m}$, com fileiras de $5 \mathrm{~m}$ espaçadas de $0,5 \mathrm{~m}$. As fileiras centrais constituíram a área experimental.

A análise de solo $(0-20 \mathrm{~cm})$ revelou os seguintes resultados: $\mathrm{P}, 9 \mathrm{mg} \mathrm{dm}^{-3}$ (resina); $\mathrm{MO}, 20 \mathrm{~g} \mathrm{dm}^{-3}$; $\mathrm{pH}\left(\mathrm{CaCl}_{2}\right), 3,7$; cátions trocáveis em mmolc $\mathrm{dm}^{-}$ ${ }^{3}$ : K, 1,2; Ca, 2; Mg, 1; H+Al, 64; $\mathrm{Al}^{3+}$, 62; soma de bases, 4; capacidade de troca catiônica, 68; saturação de bases, 6; saturação $\mathrm{Al}^{3+}$, 94; e enxofre e microelementos, em mg dm${ }^{-3}: \mathrm{S}_{-} \mathrm{SO}_{4}{ }^{2-}$, 10; B, 0,49; Cu, 1,0; Fe, 88; Mn, 8,8; e Zn, 0,5. Em outubro de 2008 foi feita calagem $\left(3\right.$ t.ha $\left.^{-1}\right) \mathrm{e}$ aplicados $80 \mathrm{~g}$ de $\mathrm{KCl}, 400 \mathrm{~g}$ de superfosfato simples e 40g FTE BR15 nas linhas de plantio em cada parcela. Com base na análise de solo, em agosto de 2009, foram aplicados, a lanço, 2,4g de ácido bórico, 40,2g de $\mathrm{KCl}, 119,6 \mathrm{~g}$ de superfosfato simples e $3.200 \mathrm{~g}$ de calcário dolomítico (PRNT 90\%) por parcela.

A avaliação durou 182 dias, de novembro/2009 a maio/2010, iniciando-se 315 dias após o plantio. $\mathrm{O}$ corte de padronização foi realizado em novembro/2009. Cada parcela foi dividida em seis subparcelas, nas quais foram feitos cortes em intervalos de 30, 60, 90 e 180 dias e cortes adicionais aos 120 e 150 dias. As subparcelas de frequência de corte de 30 dias na sombra foram capinadas acidentalmente em fevereiro/2010, de modo que foram excluídas das avaliações. A produção de MS foi avaliada a partir de duas amostras de $0,25 \mathrm{~m}^{2}$ por subparcela, cortadas a $10 \mathrm{~cm}$ (plantas eretas ou semieretas, como o Lablab) ou a $5 \mathrm{~cm}$ (plantas prostradas, como o Calopogonio) de altura do solo. As plantas foram avaliadas quanto à produção de matéria seca (MS) e valor nutricional. O material colhido foi seco em estufa a $60^{\circ} \mathrm{C}$ até peso constante, e as amostras foram avaliadas quanto ao teor de proteína bruta $(\mathrm{PB})$ e de digestibilidade in vitro 
da matéria seca (DIVMS) (Silva e Queiroz, 2002). A taxa de acúmulo $\left(\mathrm{kg} \mathrm{ha}^{-1} \mathrm{dia}^{-1}\right)$ foi calculada pela divisão da produção de matéria seca pelos dias de rebrota antes do corte. A radiação fotossinteticamente ativa foi avaliada mensalmente em oito pontos ao acaso, nas orientações geográficas $\mathrm{N}, \mathrm{S}, \mathrm{L}$ e $\mathrm{O}$, nas parcelas ao sol, e oito pontos nas parcelas sombreadas, entre $11 \mathrm{~h}$ e $12 \mathrm{~h}$, com ceptômetro (AccuPAR LP80, Decagon). Dados meteorológicos foram disponibilizados pela Estação Experimental de Ciências Florestais de Anhembi - ESALQ / USP.

Os resultados de taxa de acúmulo e de proteína foram transformados em escala logarítmica para atender às pressuposições de normalidade da distribuição dos erros e homogeneidade das variâncias. Os dados foram submetidos a análises de variância, utilizando o software SAS® (SAS 9.3, SAS Institute, Cary, North Carolina, USA), com base no modelo $Y_{i j k l m}=\mu+S_{i}+F_{j}+L_{k}+B_{l}+e_{i j k l}$, no qual $Y_{i j k l}=\operatorname{logaritmo}$ da taxa de acúmulo; $\mu=$ média geral da taxa de acúmulo; $S_{i}=$ espécie forrageira, onde $\mathrm{i}=1,2, \ldots, 9 ; F_{j}=$ frequência de corte, onde $\mathrm{j}=1,2, \ldots, 6(60,90,120,150$, e 180 dias); $L_{k}=$ iluminação, onde $\mathrm{k}=1$ e 2 (sol e sombra); $B_{l}=$ bloco, onde $1=1,2,3,4 ; e_{i j k l}=$ erro aleatório associado à taxa de acúmulo. As médias dos resultados foram comparadas pelo teste Tukey $(\mathrm{P}<0,05)$. Em seguida, foi feita a análise para cada espécie forrageira, considerando-se o mesmo modelo usado, omitindo-se o efeito da variável espécie.

\section{RESULTADOS E DISCUSSÃO}

A radiação luminosa incidente nas parcelas sombreadas $\left(252 \mu \mathrm{mol} \mathrm{m} \mathrm{m}^{-2} \mathrm{~s}^{-1}\right)$ correspondeu a $17 \%$ da radiação incidente a pleno sol $\left(1.468 \mu \mathrm{mol} \mathrm{m} \mathrm{m}^{-2} \mathrm{~s}^{-1}\right)$. Não houve deficit hídrico em dezembro e em janeiro, mas a precipitação já se mostrava insuficiente para atender à evapotranspiração a partir de fevereiro (Fig. 1).

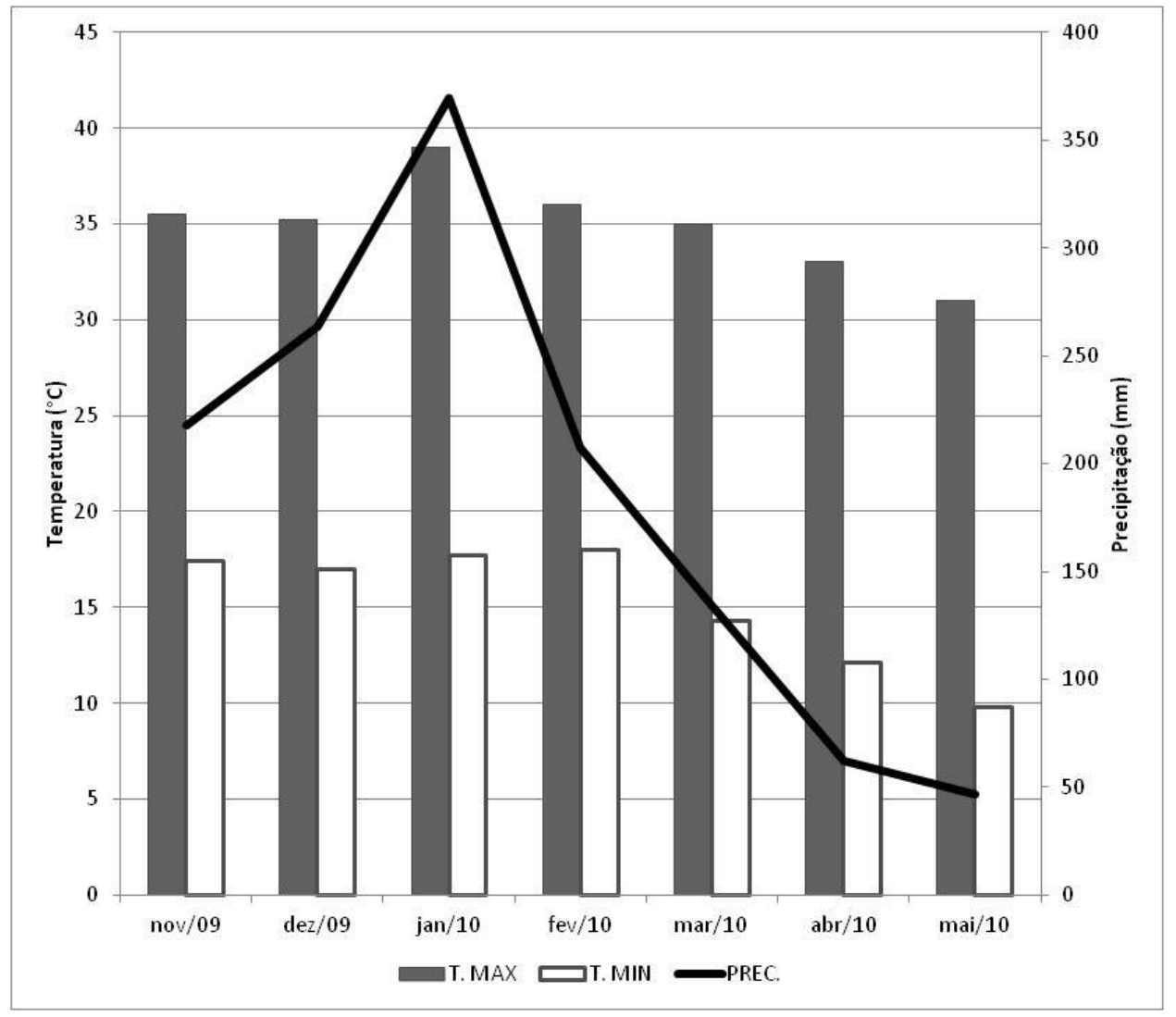

Figura 1. Temperaturas máximas e mínimas e precipitação pluvial novembro/2009 maio/2010, verificadas no município de Anhembi (SP). 
A taxa de acúmulo média (de todas as espécies) foi de $21,5 \pm 12,3 \mathrm{~kg} \cdot \mathrm{ha}^{-1} \mathrm{dia}^{-1}$ ao sol e $3,8 \pm 2,7 \mathrm{~kg} \cdot \mathrm{ha}^{-1} \mathrm{dia}^{-1}$ à sombra. A baixa disponibilidade de radiação comprometeu a produção, embora as condições hídricas e térmicas distintas associadas aos níveis de sombreamento possam ter contribuído para os resultados. Essa taxa variou entre espécies $(\mathrm{P}<0,05)$. O Estilosantes exibiu a maior taxa de acúmulo $(\mathrm{P}<0,05)$, tanto ao sol $\left(36,3 \mathrm{~kg} \cdot \mathrm{ha}^{-1} \mathrm{dia}^{-1}\right)$ quanto à sombra $\left(6,3 \mathrm{~kg} \cdot \mathrm{ha}^{-1} \mathrm{dia}^{-1}\right)$. As menores produtividades $(\mathrm{P}>0,05)$ resultaram do Lablab $\left(15,6 \mathrm{~kg} \cdot \mathrm{ha}^{-1} \mathrm{dia}^{-1}\right)$ ao sol, e de Alysicarpus $\left(1,5 \mathrm{~kg} \cdot \mathrm{ha}^{-1} \mathrm{dia}^{-1}\right)$ e de Aeschynomene $\left(2,6 \mathrm{~kg} \cdot \mathrm{ha}^{-}\right.$ ${ }^{1} \mathrm{dia}^{-1}$ ) à sombra.

Os melhores desempenhos foram verificados no meio da estação chuvosa, no corte realizado aos
120 dias, no sol e na sombra (Tab. 1). O Alysicarpus e o Aeschynomene revelaram-se pouco adaptados às condições impostas pelo sombreamento e desapareceram das parcelas. Por outro lado, a Pueraria e o Estilosantes tiveram produções acima da média em todos os cortes. Baixa produção de biomassa também foi verificada no sub-bosque de seringais asiáticos adultos semeados com Calopogonium caeruleum e Pueraria phaseoloides (Chong et al., 1997), onde a disponibilidade de forragem variou de 200 a $800 \mathrm{~kg} / \mathrm{ha}$. No Acre, contudo, A. pintoi cv. Belmonte produziu no período chuvoso, com $70 \%$ de sombra artificial, $22,4 \mathrm{~kg} \mathrm{ha}^{-1} \mathrm{dia}^{-}$ ${ }^{1}$ (Andrade et al., 2004), semelhante aos valores aqui observados ao sol com outra cultivar dessa mesma espécie.

Tabela 1. Taxa de acúmulo $\left(\mathrm{kg} \cdot \mathrm{ha}^{-1} \cdot \mathrm{dia}^{-1}\right)$ de matéria seca de oito espécies de leguminosas herbáceas tropicais plantas a pleno sol e à sombra. Anhembi (SP), novembro/2009 - maio/2010

\begin{tabular}{|c|c|c|c|c|c|c|c|}
\hline \multirow[t]{2}{*}{ Espécies } & \multicolumn{4}{|c|}{ Frequência de cortes (dias) } & \multicolumn{3}{|c|}{ Corte único (dias) } \\
\hline & 30 & 60 & 90 & 120 & 150 & 180 & Média \\
\hline Sol & 19,8 & 20,9 & 18,3 & 28,2 & 20,6 & 17,5 & 20,4 \\
\hline Lablab & $6,7 \mathrm{c}$ & $14,3 \mathrm{~b}$ & $9,7 \mathrm{bc}$ & $29,5 \mathrm{a}$ & $8,5 \mathrm{bc}$ & $11,1 \mathrm{bc}$ & 11,1 \\
\hline Centrosema & $19,5 \mathrm{ab}$ & $19,3 \mathrm{ab}$ & $18,3 \mathrm{ab}$ & $23,2 \mathrm{a}$ & $13,8 \mathrm{ab}$ & $11,5 \mathrm{~b}$ & 18,5 \\
\hline Clitoria & $19,1 \mathrm{a}$ & $21,2 \mathrm{a}$ & $17,4 \mathrm{a}$ & $27,1 \mathrm{a}$ & $16,2 \mathrm{a}$ & $21,2 \mathrm{a}$ & 19,9 \\
\hline Pueraria & $23,3 \mathrm{a}$ & $27,2 \mathrm{a}$ & $22,0 \mathrm{a}$ & $22,9 \mathrm{a}$ & $17,8 \mathrm{a}$ & $16,9 \mathrm{a}$ & 23,1 \\
\hline Alysicarpus & $16,3 \mathrm{~b}$ & $15,8 \mathrm{~b}$ & $14,3 \mathrm{~b}$ & $31,6 \mathrm{a}$ & $15,3 \mathrm{ab}$ & $14,9 \mathrm{ab}$ & 16,8 \\
\hline Aeschynomene & $21,5 \mathrm{a}$ & $21,2 \mathrm{a}$ & 17,6 a & $27,9 \mathrm{a}$ & $21,0 \mathrm{a}$ & $19,3 \mathrm{a}$ & 21,1 \\
\hline Estilosantes & $28,8 \mathrm{c}$ & $32,8 \mathrm{bc}$ & $35,6 \mathrm{abc}$ & $55,4 \mathrm{ab}$ & $65,2 \mathrm{a}$ & $45,5 \mathrm{abc}$ & 36,3 \\
\hline Calopogonio & $17,3 \mathrm{a}$ & $15,5 \mathrm{a}$ & $11,2 \mathrm{a}$ & $17,7 \mathrm{a}$ & $11,8 \mathrm{a}$ & 8,0 a & 15,0 \\
\hline Arachis & $23,7 \mathrm{a}$ & $21,1 \mathrm{ab}$ & $18,8 \mathrm{ab}$ & $18,6 \mathrm{ab}$ & $15,4 \mathrm{bc}$ & $9,2 \mathrm{c}$ & 20,5 \\
\hline Sombra & & 2,6 & 2,4 & 3,9 & 2,8 & 1,7 & 2,9 \\
\hline Lablab & - & $1,6 \mathrm{~b}$ & $1,5 \mathrm{ab}$ & $3,6 \mathrm{a}$ & $1,5 \mathrm{ab}$ & $0,9 \mathrm{~b}$ & 2,4 \\
\hline Centrosema & - & $3,6 \mathrm{a}$ & $3,9 \mathrm{a}$ & $3,9 \mathrm{a}$ & $3,1 \mathrm{ab}$ & $1,3 \mathrm{~b}$ & 3,8 \\
\hline Clitoria & - & $0,9 \mathrm{~b}$ & $1,8 \mathrm{~b}$ & $4,1 \mathrm{a}$ & $2,3 \mathrm{ab}$ & $2,2 \mathrm{ab}$ & 1,9 \\
\hline Pueraria & - & $5,0 \mathrm{a}$ & $4,2 \mathrm{a}$ & $5,4 \mathrm{a}$ & $5,1 \mathrm{a}$ & $3,1 \mathrm{~b}$ & 4,6 \\
\hline Alysicarpus & - & 0,1 & 0,0 & 0,0 & 0,0 & 0,0 & 0,0 \\
\hline Aeschynomene & - & $0,6 \mathrm{a}$ & $0,0 \mathrm{a}$ & $0,2 \mathrm{a}$ & $0,0 \mathrm{a}$ & $0,3 \mathrm{a}$ & 0,8 \\
\hline Estilosantes & - & $4,3 \mathrm{~b}$ & $4,1 \mathrm{~b}$ & $9,1 \mathrm{a}$ & $6,7 a b$ & $5,2 \mathrm{ab}$ & 5,6 \\
\hline Calopogonio & - & $3,7 \mathrm{a}$ & $3,1 \mathrm{a}$ & $3,9 \mathrm{a}$ & $2,7 \mathrm{a}$ & $1,5 \mathrm{a}$ & 3,6 \\
\hline Arachis & - & $3,3 \mathrm{bc}$ & $3,3 \mathrm{bc}$ & $5,3 \mathrm{a}$ & $3,9 a b$ & $2,1 \mathrm{c}$ & 3,8 \\
\hline
\end{tabular}

Em cada espécie e frequência de corte, valores seguidos por letras diferentes indicam diferenças pelo teste Tukey $(\mathrm{P}<0,05)$

É importante conhecer a estabilidade da produção de MS nos cortes (Tab. 2) para estabelecer as estratégias de manejo e a necessidade de suplementação. Ao sol, somente a produção de MS do Lablab foi estável na frequência de corte de 30 dias $(\mathrm{P}>0,05)$, embora tenha decrescido de março em diante. As demais espécies apresentaram pico de produção em fevereiro e reagiram de forma distinta aos cortes; ao contrário da Centrosema, da Clitoria e do
Alysicarpus, a taxa de acúmulo pouco variou no caso do Estilosantes ao sol. Nas avaliações aos 60 dias ao sol, as produções foram mais altas em março. A Clitoria e o Arachis mostraram as maiores flutuações. Na frequência de corte de 90 dias ao sol não houve diferença na produção do Lablab, da Centrosema, da Pueraria e do Estilosantes. Nas demais, a produção foi menor ao segundo corte, com médias de $21 \%$ a $56 \%$ daquelas obtidas em fevereiro. A redução das 
taxas de acúmulo com o avançar do período chuvoso devem-se à associação de variáveis climáticas (redução da umidade, da temperatura e do fotoperíodo), reprodução (realocação de recursos, surgimento de novos drenos) e senescência expressa pela redução da taxa de crescimento, morte de tecidos e perda de folhas (Villarreal et al., 2005).

Tabela 2. Médias de taxa de acúmulo $\left(\mathrm{kg} . \mathrm{ha}^{-1}\right.$. dia $\left.^{-1}\right)$ por corte, nas frequências de corte de 30,60 e 90 dias, de nove espécies de leguminosas herbáceas tropicais cultivadas a pleno sol. Anhembi (SP), novembro/2009 - maio/2010

\begin{tabular}{|c|c|c|c|c|c|c|}
\hline \multirow[t]{2}{*}{ Forrageiras } & \multicolumn{6}{|l|}{30 dias } \\
\hline & Dezembro & Janeiro & Fevereiro & Março & Abril & Maio \\
\hline Lablab & $13,8 \mathrm{a}$ & $8,4 \mathrm{a}$ & $11,4 \mathrm{a}$ & $1,9 \mathrm{a}$ & $1,8 \mathrm{a}$ & $1,6 \mathrm{a}$ \\
\hline Centrosema & $14,6 \mathrm{bcd}$ & $23,2 \mathrm{ab}$ & $34,2 \mathrm{a}$ & $13,2 \mathrm{~cd}$ & $23,6 \mathrm{abc}$ & $8,8 \mathrm{~d}$ \\
\hline Clitoria & $30,9 \mathrm{ab}$ & $17,7 \mathrm{abc}$ & $31,1 \mathrm{a}$ & $20,2 \mathrm{ab}$ & $7,2 \mathrm{~cd}$ & $3,9 \mathrm{~d}$ \\
\hline Pueraria & $9,4 \mathrm{c}$ & $20,3 \mathrm{~b}$ & $42,2 \mathrm{a}$ & $25,4 \mathrm{ab}$ & $22,6 \mathrm{ab}$ & $20,0 \mathrm{~b}$ \\
\hline Alysicarpus & $19,6 b c$ & $8,6 \mathrm{c}$ & $33,9 \mathrm{a}$ & $27,3 \mathrm{ab}$ & $9,6 \mathrm{abc}$ & $0,0 \mathrm{~d}$ \\
\hline Aeschynomene & $28,5 \mathrm{ab}$ & $13,1 \mathrm{bc}$ & $31,2 \mathrm{a}$ & $32,5 \mathrm{a}$ & $15,0 \mathrm{c}$ & $8,5 \mathrm{c}$ \\
\hline Estilosantes & $17,2 \mathrm{~b}$ & $21,1 \mathrm{~b}$ & $53,8 \mathrm{a}$ & $33,9 \mathrm{ab}$ & $28,0 \mathrm{ab}$ & $18,7 \mathrm{~b}$ \\
\hline Calopogonio & $27,8 \mathrm{ab}$ & $10,6 \mathrm{c}$ & $28,9 \mathrm{a}$ & $9,5 \mathrm{bc}$ & $18,4 \mathrm{abc}$ & $8,2 \mathrm{c}$ \\
\hline \multirow[t]{3}{*}{ Arachis } & $28,1 \mathrm{ab}$ & $22,3 \mathrm{abc}$ & $35,0 \mathrm{a}$ & $19,9 \mathrm{bc}$ & $21,0 \mathrm{bc}$ & $16,1 \mathrm{c}$ \\
\hline & 60 dias & & & 90 dias & & \\
\hline & Janeiro & Março & Maio & Fevereiro & \multicolumn{2}{|c|}{ Maio } \\
\hline Lablab & $15,0 \mathrm{~b}$ & $23,4 \mathrm{a}$ & $4,5 \mathrm{~b}$ & $12,8 \mathrm{a}$ & \multicolumn{2}{|c|}{$6,5 \mathrm{a}$} \\
\hline Centrosema & $21,2 \mathrm{a}$ & $22,5 \mathrm{a}$ & $14,0 \mathrm{a}$ & $20,3 \mathrm{a}$ & \multicolumn{2}{|c|}{$16,3 \mathrm{a}$} \\
\hline Clitoria & $24,5 \mathrm{~b}$ & $35,8 \mathrm{a}$ & $3,2 \mathrm{c}$ & $28,7 \mathrm{a}$ & \multicolumn{2}{|c|}{$6,1 \mathrm{~b}$} \\
\hline Pueraria & $15,1 \mathrm{~b}$ & $37,8 \mathrm{a}$ & $28,8 \mathrm{a}$ & $19,5 \mathrm{a}$ & \multicolumn{2}{|c|}{$24,5 \mathrm{a}$} \\
\hline Alysicarpus & $22,1 \mathrm{a}$ & $22,8 \mathrm{a}$ & $2,4 \mathrm{a}$ & 28,5 & \multicolumn{2}{|c|}{0,0} \\
\hline Aeschynomene & $22,2 \mathrm{ab}$ & $25,9 \mathrm{a}$ & $15,6 \mathrm{~b}$ & $24,3 \mathrm{a}$ & \multicolumn{2}{|c|}{$10,9 \mathrm{~b}$} \\
\hline Estilosantes & $19,0 \mathrm{~b}$ & $57,1 \mathrm{a}$ & $22,3 \mathrm{~b}$ & $32,5 \mathrm{a}$ & \multicolumn{2}{|c|}{$38,7 \mathrm{a}$} \\
\hline Calopogonio & $19,5 \mathrm{a}$ & $18,8 \mathrm{a}$ & $8,2 \mathrm{~b}$ & $16,8 \mathrm{a}$ & \multicolumn{2}{|c|}{$5,5 \mathrm{~b}$} \\
\hline Arachis & $22,5 \mathrm{~b}$ & $30,3 \mathrm{a}$ & $10,4 \mathrm{c}$ & $27,2 \mathrm{a}$ & \multicolumn{2}{|c|}{$10,5 \mathrm{~b}$} \\
\hline
\end{tabular}

Em cada espécie e frequência de corte, valores seguidos por letras diferentes indicam diferenças pelo teste Tukey $(\mathrm{P}<0,05)$.

As produções da Centrosema e da Clitoria na frequência de corte de 60 dias foram estáveis na sombra (Tab. 3), com maior flutuação do Calopogonio. O Lablab não persistiu nas frequências de corte de 60 e de 90 dias. A Centrosema, a Pueraria, o Estilosantes, o Calopogonio e o Arachis tiveram produção acima da média nas duas frequências de corte.

Tabela 3. Médias de taxa de acúmulo $\left(\mathrm{kg} . \mathrm{ha}^{-1} \cdot \mathrm{dia}^{-1}\right)$ por corte, nas frequências de corte de 60 e 90 dias, de nove espécies de leguminosas tropicais herbáceas cultivadas à sombra. Anhembi (SP), novembro/2009 - maio/2010

\begin{tabular}{llllll}
\hline Forrageiras & \multicolumn{3}{c}{ 60 dias } & & \multicolumn{3}{c}{90 dias } \\
\cline { 2 - 6 } & Janeiro & Março & Maio & Fevereiro & Maio \\
\hline Lablab & 4,9 & 0,0 & 0,0 & 2,8 & 0,2 \\
Centrosema & $2,8 \mathrm{a}$ & $5,1 \mathrm{a}$ & $2,8 \mathrm{a}$ & $3,8 \mathrm{a}$ & $4,0 \mathrm{a}$ \\
Clitoria & $1,4 \mathrm{a}$ & $0,8 \mathrm{a}$ & $0,3 \mathrm{a}$ & $3,0 \mathrm{a}$ & $0,1 \mathrm{~b}$ \\
Pueraria & $3,8 \mathrm{~b}$ & $6,4 \mathrm{a}$ & $4,8 \mathrm{ab}$ & $5,1 \mathrm{a}$ & $3,3 \mathrm{~b}$ \\
Alysicarpus & 0,2 & 0,0 & 0,0 & 0,0 & 0,0 \\
Aeschynomene & $1,5 \mathrm{a}$ & 0,00 & $0,2 \mathrm{a}$ & 0,1 & 0,0 \\
Estilosantes & $5,4 \mathrm{a}$ & $5,6 \mathrm{a}$ & $1,8 \mathrm{~b}$ & $5,6 \mathrm{a}$ & $2,6 \mathrm{~b}$ \\
Calopogonio & $7,0 \mathrm{a}$ & $3,2 \mathrm{~b}$ & $0,8 \mathrm{c}$ & $5,1 \mathrm{a}$ & $1,1 \mathrm{~b}$ \\
Arachis & $3,9 \mathrm{a}$ & $4,2 \mathrm{a}$ & $1,1 \mathrm{~b}$ & $4,8 \mathrm{a}$ & $1,7 \mathrm{~b}$ \\
\hline
\end{tabular}

Letras diferentes, nas linhas, por espécie e frequência de corte, indicam diferenças entre as médias pelo teste Tukey $(\mathrm{P}<0,05)$. 
Os teores de PB (Tab. 4) e de DIVMS (Tab. 5) variaram com a frequência de corte $(\mathrm{P}<0,05)$. Ao sol, os cortes de 30 dias apresentavam valores elevados de proteína bruta e de digestibilidade, enquanto aos 180 dias a qualidade das forrageiras decaía consideravelmente. Com o avanço da estação chuvosa, algumas leguminosas começaram a perder folhas e secar, situação muito evidente no Alysicarpus, o que explica o acentuado declínio da digestibilidade observado, atingindo $26,3 \%$.

À sombra, observaram-se as concentrações mais elevadas de proteína aos 90 dias em todas as forrageiras, exceto na Aeschynomene, cujo ápice verificou-se aos 120 dias, e no Alysicarpus, cuja produção de matéria seca, entretanto, foi insignificante. A digestibilidade à sombra mostrou-se elevada aos 60 e aos 90 dias para grande parte das forrageiras, novamente com exceção da Aeschynomene e da Alysiscarpus.

Os teores de proteína bruta das plantas cultivadas na sombra foram superiores aos das plantas cultivadas ao sol (Tab. 4). Embora a digestibilidade tenha se mostrado superior sob o sol no caso da Pueraria, com padrão inverso no caso das plantas de Lablab e de Estilosantes, o efeito das condições locais de iluminação foi menos acentuado para digestibilidade da matéria seca nas demais espécies (Tab. 5).

Verificou-se correlação positiva e significativa $(\mathrm{P}<0,05)$ entre a digestibilidade e a proteína para a maior parte das espécies, exceto o Calopogonio ao sol $(\mathrm{P}>0,05)$ e a Centrosema e a Pueraria na sombra $(\mathrm{P}>0,05)$. A correlação entre a taxa de acúmulo e a proteína bruta foi negativa para o Lablab, o Estilosantes e o Arachis ao sol $(\mathrm{P}<0,05)$ e para a Centrosema, a Pueraria e o Estilosantes à sombra $(\mathrm{P}<0,05)$, sendo não significativa $(\mathrm{P}>0,05)$ para as demais. A digestibilidade decresceu com a taxa de acúmulo para o Estilosantes e o Arachis ao sol $(\mathrm{P}<0,05)$ e para a Clitoria e o Estilosantes à sombra $(\mathrm{P}<0,05)$, sendo não significativa $(\mathrm{P}>0,05)$ para as demais. Essas observações talvez possam ser explicadas por alterações na relação folha/talo associadas ao desenvolvimento das plantas.

Tabela 4. Teor médio de proteína bruta $(\%)$ por espécie e frequência de corte, ao sol e à sombra, de nove espécies de leguminosas tropicais herbáceas. Anhembi (SP), novembro/2009 - maio/2010

\begin{tabular}{|c|c|c|c|c|c|c|}
\hline \multicolumn{7}{|c|}{ Frequência de cortes } \\
\hline & 30 dias & 60 dias & 90 dias & 120 dias & 150 dias & 180 dias \\
\hline \multicolumn{7}{|l|}{ Sol } \\
\hline Lablab & $24,9 \mathrm{a}$ & $19,3 \mathrm{~b}$ & $20,6 \mathrm{~b}$ & $17,9 \mathrm{bc}$ & $20,1 \mathrm{~b}$ & $14,4 \mathrm{c}$ \\
\hline Centrosema & $26,7 \mathrm{a}$ & $23,7 \mathrm{c}$ & $25,4 \mathrm{~b}$ & $23,1 \mathrm{c}$ & $27,0 \mathrm{a}$ & $23,2 \mathrm{c}$ \\
\hline Clitoria & $25,6 \mathrm{a}$ & $18,6 \mathrm{c}$ & $19,9 \mathrm{~b}$ & $18,3 \mathrm{c}$ & $16,2 \mathrm{~d}$ & $14,1 \mathrm{e}$ \\
\hline Pueraria & $28,4 \mathrm{a}$ & $19,1 \mathrm{c}$ & $21,4 \mathrm{~b}$ & $19,9 \mathrm{bc}$ & $22,9 \mathrm{~b}$ & $20,3 \mathrm{bc}$ \\
\hline Alysicarpus & $21,3 \mathrm{a}$ & $19,0 \mathrm{~b}$ & $17,5 \mathrm{c}$ & $12,9 \mathrm{~d}$ & $11,0 \mathrm{e}$ & $8,2 \mathrm{f}$ \\
\hline Aeschynomene & $23,4 \mathrm{a}$ & $17,4 \mathrm{~b}$ & $23,7 \mathrm{a}$ & $16,7 \mathrm{~b}$ & $17,9 \mathrm{~b}$ & $13,6 \mathrm{~b}$ \\
\hline Estilosantes & $19,9 \mathrm{a}$ & $13,2 \mathrm{~cd}$ & $16,2 \mathrm{~b}$ & $13,8 \mathrm{c}$ & $12,8 \mathrm{~cd}$ & $11,7 \mathrm{~d}$ \\
\hline Calopogonio & $22,7 \mathrm{ab}$ & $20,6 \mathrm{~cd}$ & $20,9 \mathrm{bc}$ & $20,5 \mathrm{~cd}$ & $23,7 \mathrm{a}$ & $19,5 \mathrm{~d}$ \\
\hline Arachis & $22,7 \mathrm{a}$ & $19,7 \mathrm{~b}$ & $17,9 \mathrm{c}$ & $16,5 \mathrm{~cd}$ & $16,4 \mathrm{~cd}$ & $14,6 \mathrm{~d}$ \\
\hline \multicolumn{7}{|l|}{ Sombra } \\
\hline Lablab & - & $21,5 \mathrm{~b}$ & $27,8 \mathrm{a}$ & $24,6 \mathrm{ab}$ & $24,0 \mathrm{~b}$ & $22,1 \mathrm{~b}$ \\
\hline Centrosema & - & $23,1 \mathrm{c}$ & $25,8 \mathrm{a}$ & $23,9 \mathrm{~b}$ & $23,5 \mathrm{bc}$ & $24,6 \mathrm{ab}$ \\
\hline Clitoria & - & $19,4 \mathrm{~b}$ & $22,7 \mathrm{a}$ & $18,0 \mathrm{~b}$ & $18,9 \mathrm{~b}$ & $16,2 \mathrm{c}$ \\
\hline Pueraria & - & $18,8 \mathrm{~b}$ & $21,8 \mathrm{a}$ & $19,4 \mathrm{~b}$ & $19,5 \mathrm{~b}$ & $18,8 \mathrm{~b}$ \\
\hline Alysicarpus & - & - & - & - & - & - \\
\hline Aeschynomene & - & $16,9 \mathrm{c}$ & $20,2 b$ & $20,9 \mathrm{a}$ & $12,7 \mathrm{e}$ & $15,0 \mathrm{~d}$ \\
\hline Estilosantes & - & $15,9 \mathrm{a}$ & $16,1 \mathrm{a}$ & $15,5 \mathrm{a}$ & $13,3 \mathrm{~b}$ & $12,8 \mathrm{~b}$ \\
\hline Calopogonio & - & $19,0 \mathrm{c}$ & $22,5 \mathrm{a}$ & $23,0 \mathrm{a}$ & $20,9 \mathrm{~b}$ & $20,9 \mathrm{~b}$ \\
\hline Arachis & - & $19,0 \mathrm{ab}$ & $19,8 \mathrm{ab}$ & $20,5 \mathrm{a}$ & $17,2 \mathrm{c}$ & $16,5 \mathrm{c}$ \\
\hline
\end{tabular}

Letras diferentes, nas linhas, por espécie e local, indicam diferenças entre as médias pelo teste Tukey $(\mathrm{P}<0,05)$. 
Tabela 5. Digestibilidade in vitro da matéria seca (\%) por espécie e frequência de corte, ao sol e à sombra, de nove espécies de leguminosas tropicais herbáceas, cultivadas a pleno sol e sob sombra. Anhembi (SP), novembro/2009 - maio/2010

\begin{tabular}{|c|c|c|c|c|c|c|}
\hline \multicolumn{7}{|c|}{ Frequência de cortes } \\
\hline & 30 dias & 60 dias & 90 dias & 120 dias & 150 dias & 180 dias \\
\hline \multicolumn{7}{|l|}{ Sol } \\
\hline Lablab & $72,5 \mathrm{a}$ & $65,2 b$ & $59,8 \mathrm{c}$ & $66,5 \mathrm{ab}$ & $59,0 \mathrm{bc}$ & $47,2 \mathrm{~d}$ \\
\hline Centrosema & 53,9 a & $51,4 \mathrm{a}$ & $46,3 \mathrm{~b}$ & $39,6 \mathrm{c}$ & $44,8 \mathrm{~b}$ & $45,3 \mathrm{~b}$ \\
\hline Clitoria & $66,1 \mathrm{a}$ & $60,6 b$ & $58,1 \mathrm{c}$ & $52,9 \mathrm{~d}$ & 46,9 e & $39,8 \mathrm{f}$ \\
\hline Pueraria & $64,4 \mathrm{a}$ & $57,1 \mathrm{~b}$ & $56,0 \mathrm{~cd}$ & $53,6 \mathrm{~d}$ & $56,4 \mathrm{bc}$ & $53,1 \mathrm{~d}$ \\
\hline Alysicarpus & $64,8 \mathrm{a}$ & $61,6 \mathrm{a}$ & $52,4 \mathrm{~b}$ & $44,2 \mathrm{c}$ & $30,8 \mathrm{~d}$ & $26,3 \mathrm{~d}$ \\
\hline Aeschynomene & $66,3 \mathrm{a}$ & $54,4 \mathrm{bc}$ & $57,7 \mathrm{~b}$ & $52,3 \mathrm{c}$ & $50,7 \mathrm{c}$ & $42,5 \mathrm{~d}$ \\
\hline Estilosantes & $62,1 \mathrm{a}$ & $56,0 \mathrm{~b}$ & $56,7 \mathrm{~b}$ & $48,6 \mathrm{c}$ & $47,0 \mathrm{c}$ & $41,7 \mathrm{~d}$ \\
\hline Calopogonio & $51,8 \mathrm{a}$ & $48,6 \mathrm{~b}$ & $43 \mathrm{c}$ & $44,7 \mathrm{c}$ & $43,7 \mathrm{c}$ & $40,0 \mathrm{~d}$ \\
\hline Arachis & $66,7 \mathrm{a}$ & $65,8 \mathrm{a}$ & $61,8 \mathrm{~b}$ & $59,4 \mathrm{c}$ & $62,0 \mathrm{~b}$ & $58,2 \mathrm{c}$ \\
\hline \multicolumn{7}{|l|}{ Sombra } \\
\hline Lablab & - & $72,4 \mathrm{a}$ & $70,1 \mathrm{a}$ & $63,0 \mathrm{~b}$ & $56,8 \mathrm{c}$ & $59,7 \mathrm{bc}$ \\
\hline Centrosema & - & $54,7 \mathrm{a}$ & $51,1 \mathrm{~b}$ & $47,5 \mathrm{c}$ & $41,8 \mathrm{~d}$ & 49, bc \\
\hline Clitoria & - & $60,7 \mathrm{a}$ & 62,9 a & $52,3 \mathrm{~b}$ & $53,1 \mathrm{~b}$ & $48,4 \mathrm{c}$ \\
\hline Pueraria & - & $59,2 \mathrm{a}$ & $51,2 \mathrm{a}$ & $48,2 \mathrm{c}$ & $46,5 \mathrm{c}$ & $52,6 \mathrm{~b}$ \\
\hline Alysicarpus & - & - & - & - & - & - \\
\hline Aeschynomene & - & $59,7 \mathrm{c}$ & $46,8 \mathrm{~d}$ & $62,4 \mathrm{~b}$ & $64,3 \mathrm{a}$ & $46,2 \mathrm{e}$ \\
\hline Estilosantes & - & $60,4 \mathrm{a}$ & $56,7 \mathrm{a}$ & $54,3 \mathrm{~b}$ & $45,2 \mathrm{~d}$ & $51,2 \mathrm{c}$ \\
\hline Calopogonio & - & $45,6 \mathrm{a}$ & $43,3 \mathrm{ab}$ & $42,5 \mathrm{ab}$ & $34,2 \mathrm{c}$ & $42,3 \mathrm{~b}$ \\
\hline Arachis & - & $66,1 \mathrm{a}$ & $63,6 \mathrm{~b}$ & $57,7 \mathrm{c}$ & $57,0 \mathrm{c}$ & $59,6 \mathrm{c}$ \\
\hline
\end{tabular}

Letras diferentes, nas linhas, por espécie e local, indicam diferenças entre as médias pelo teste Tukey $(\mathrm{P}<0,05)$.

A produtividade média pode mascarar o desempenho que pode ser obtido com a utilização da frequência de corte apropriada. A altura de corte, juntamente com a frequência, é importante variável para definição da produtividade (Ferreira et al., 2010). Neste estudo, optou-se por manter uma única altura de corte, estabelecida em função do hábito de crescimento; contudo, a combinação resultante não foi a ideal para todas as espécies avaliadas. A produção média do Arachis em todos os cortes superou a média das leguminosas, com $20,5 \mathrm{~kg} \cdot \mathrm{ha}^{-1} \cdot \mathrm{dia}^{-1}$ ao sol e $3,8 \mathrm{~kg} \cdot \mathrm{ha}^{-1} \cdot \mathrm{dia}^{-1}$ à sombra, e se reduziu a partir de março (Tab. 2 e 3). Nessa época, observou-se a associação de temperaturas abaixo de $15-17^{\circ} \mathrm{C}$ e de restrição hídrica (Fig. 1), limitando seu crescimento (Fonseca e Martuscello, 2010). Recomenda-se que Arachis pintoi seja manejado com três a cinco cortes baixos na estação chuvosa (Fonseca e Martuscello, 2010). O melhor desempenho do Arachis foi obtido na frequência de corte de 30 dias ao sol (Tab. 1). Villarreal et al. (2005) e Urbano et al. (2010) relataram maior produção de MS para Arachis pintoi com menor frequência de cortes; porém, uma das variedades estudadas por Urbano et al. (2010) aumentou a produção quando a frequência de corte passou de 49 dias para 35 dias. Embora Arachis spp. seja tolerante ao sombreamento, a frequência de corte deve ser reduzida na sombra intensa: a menor radiação compromete a produção de carboidratos e a recuperação das reservas da planta após a desfolha, o que explica por que a taxa de acúmulo do Arachis foi semelhante aos 60 dias e aos 90 dias (Tab. 1), mas inferior àquela obtida nos cortes de 120 ou de 150 dias. Ferreira et al. (2010) também relataram maior produção na sombra com maiores intervalos de corte. Baixas frequências de corte prestam-se ao diferimento de pastagem, já que o Arachis apresenta altos teores de proteína e de digestibilidade, sofrendo pequenas variações com a idade (Fonseca e Martuscello, 2010). Os teores de proteína do Arachis variaram de $22,7 \%$ na frequência de corte de 30 dias a $14,6 \%$, aos 180 dias ao sol; e de $20,5 \%$ aos 120 dias a $16,5 \%$ aos 180 dias à sombra, próximos à faixa relatada por Fonseca e Martuscello (2010). A proteína foi reduzida com o aumento do intervalo de corte $(\mathrm{P}<0,05$; Tab. 4$)$, de modo análogo ao observado por Villarreal $e t$ al. (2005). A digestibilidade é elevada, mesmo tendo decrescido $(\mathrm{P}<0,05)$ com a menor 
frequência de corte, ficando próxima aos $60 \%$ $70 \%$ relatados por Cook et al.(2005).

O Estilosantes destacou-se ao sol e à sombra, mas não respondeu bem à maior frequência de corte. Teve o pior desempenho com cortes a cada 30 dias ao sol, possivelmente devido à maior perda dos meristemas apicais resultante do seu hábito de crescimento semiereto. Ao sol, pode ser recomendado o corte aos 90 dias, que produziu $6.408 \mathrm{~kg}$ com $16,2 \%$ de PB e $56,7 \%$ de DIVMS. Embora a produção aumente em frequências de corte menores, a digestibilidade alcançou valores inferiores a 55\%. À sombra não houve diferença entre as frequências de corte de 60 e de 90 dias, cujas taxas de acúmulo foram inferiores às demais. A maior produtividade na sombra foi obtida aos 120 dias, com $9,11 \mathrm{~kg}$. ha1.dia-1, $15,5 \%$ de PB e $54,3 \%$ de DIVMS. Devido a essas características, o Estilosantes à sombra pode ser recomendado para pastejo diferido. A produção em Mato Grosso do Sul atinge $14.000 \mathrm{~kg} \mathrm{ha}^{-1}$.ano, com PB de $12 \%$ a $18 \%$ e DIVMS de $55 \%$ a $60 \%$ (Fonseca e Martuscello, 2010).

O Calopogonio é uma espécie estacional, com produção superior a $3.000 \mathrm{~kg} \cdot \mathrm{ha}^{-1}$ no período chuvoso, considerado pouco tolerante a ambientes com interceptação superior a $60 \%$ da luz incidente. Recomenda-se que se evite a desfolha severa, mantendo altura de corte de 20 $\mathrm{cm}$ e intervalo de corte de 90 a 120 dias (Fonseca e Martuscello, 2010). Neste experimento, o Calopogonio apresentou taxas de acúmulo semelhantes em todas as frequências de corte, ao sol e à sombra. A produção média de Calopogonio alcançou 3.114kg.ha ${ }^{-1}$ com $22,7 \%$ de PB e $51,8 \%$ de DIVMS nos cortes a cada 30 dias, a $5 \mathrm{~cm}$ de altura, ao sol, contrastando com a recomendação acima. O Calopogonio teve baixa produção na sombra (270kg.ha-1 em 180 dias), ainda que seu desempenho se mostrasse superior na sombra ao de Lablab, de Clitoria, de Alysicarpus e de Aeschynomene. A PB varia de $16 \%$ a $24 \%$ e a DIVMS, de $58 \%$ a $66 \%$, com menor valor para intervalos de corte mais longos. A baixa digestibilidade pode ser atribuída à densa pilosidade que recobre suas folhas e caules (Fonseca e Martuscello, 2010). Os resultados aqui encontrados (Tab. 4 e 5) estão na faixa relatada acima.
Não houve diferença na produtividade da Centrosema entre as frequências de corte de 30 , 60 e 90 dias ao sol, que se mantiveram elevadas, e entre 60, 90, 120 e 150 dias à sombra. A produção ao sol é compatível com produções de $4.950 \mathrm{~kg} / \mathrm{ha}^{-1}$.ano ${ }^{-1}$ relatadas pela FAO (2009). A Centrosema persistiu sob desfolha intensa, ainda que seja indicada a altura de corte de 37,5 a 45 cm (FAO, 2009). As leguminosas volúveis são mais sensíveis a desfolhas frequentes e severas que plantas prostradas, devido à maior remoção dos pontos de crescimento (Andrade et al., 2004). A produção da Centrosema foi inferior apenas nos cortes aos 180 dias, ao sol e à sombra (Tab. 1), reflexo de mudanças ambientais e senescência, entre outros. A PB (Tab. 4) aproximou-se dos valores registrados para a espécie (Cook et al., 2005). A DIVMS variou de $40 \%$ a $54 \%$, com valores mais elevados nos cortes de 30 dias e de 60 dias. Há relatos de digestibilidade em torno de $53 \%$ para a espécie (Cook et al., 2005; FAO, 2009). As produtividades e a qualidade alcançadas por essa espécie no presente experimento indicam que o uso das maiores frequências de corte é mais apropriado.

O Lablab é uma espécie anual ou perene de ciclo curto, e o manejo adequado pode assegurar melhor aproveitamento da produção residual. Embora essa espécie tenha apresentado o pior desempenho médio ao sol $(15,6 \mathrm{~kg}$ ha-1 dia-1, $43 \%$ de Estilosantes), sua produção dobrou com o corte aos 120 dias $\left(29,5 \mathrm{~kg} \mathrm{ha}^{-1} \mathrm{dia}^{-1}\right)$, com $17,9 \%$ de PB e $66,5 \%$ de DIVMS, o que indica possibilidade de seu uso em sistema de pastejo diferido. À sombra, a produção de MS também foi mais elevada aos 120 dias, com PB em $24 \%$ e DIVMS de $63 \%$. Embora tenha sido recomendo para essa espécie pastejo leve, com altura de corte acima de $25 \mathrm{~cm}$ para remoção apenas das folhas (Fonseca e Martuscello, 2010), o corte mais severo não comprometeu a produção, já que, dentro de cada frequência de corte, não houve diferença (Tab. 2) para os cortes aos 30 dias e 90 dias, ao sol. A produção do Lablab foi insignificante após o primeiro corte na sombra.

A produtividade da Pueraria na estação chuvosa a colocou dentre as forrageiras de melhor desempenho nas condições deste experimento, com 23,3kg.ha ${ }^{-1} \cdot$ dia $^{-1}$ ao sol (4.192kg.ha $\left.{ }^{-1}\right)$ e $4,7 \mathrm{~kg} \cdot \mathrm{ha}^{-1}$. dia ${ }^{-1}$ à sombra. Há relatos de produtividade entre $4.180 \mathrm{~kg}$ (FAO, 2009) e 
15.000kg.ha ${ }^{-1}$ (Fonseca e Martuscello, 2010). Na Austrália, produziram-se $9.607 \mathrm{~kg} \cdot \mathrm{ha}^{-1}$ em três cortes na estação chuvosa (FAO, 2009). Por ser volúvel, a desfolha a $10 \mathrm{~cm}$ deve ter removido a camada superior de talos e folhas jovens, tecidos mais fotossinteticamente ativos, o que levaria a uma fase de recuperação relativamente longa a partir dos meristemas inferiores. Contudo, a Pueraria não mostrou diferenças na produção entre frequências de corte ao sol; à sombra, a produção foi menor no corte aos 180 dias (Tab. 1). A PB e a DIVMS (Tab. 4 e 5) foram semelhantes aos relatados para a espécie (FAO, 2009; Cook et al., 2005; Fonseca e Martuscello, 2010). Cortes aos 30, 60, 90 ou 150 dias, ao sol, e aos 60 dias, na sombra, podem ser indicados para a Pueraria.

A Clitoria apresentou, em média, $19,9 \mathrm{~kg} \cdot \mathrm{ha}^{-1}$. $\mathrm{dia}^{-1}$ ao sol no verão $\left(3.577 \mathrm{~kg} \cdot \mathrm{ha}^{-1}\right)$, e $2,9 \mathrm{~kg} \cdot \mathrm{ha}^{-1}$. $\operatorname{dia}^{-1}$ à sombra. Não houve diferença de desempenho entre as frequências de corte ao sol, mas a qualidade nutricional (Tab. 4 e 5) foi superior nos cortes de 30, 60 e 90 dias, apoiando a recomendação de que a Clitoria seja submetida a intervalo de corte de 56 dias sob pastejo rotacionado (Cook et al., 2005). À sombra as melhores respostas foram obtidas com menor frequência de corte (120, 150 e 180 dias), mas a digestibilidade nesses cortes foi mais baixa $(48,4 \%$ a $53,0 \%)$, sugerindo a escolha de outras espécies para compor o sub-bosque. A PB e a DIVMS da forragem produzida situaram-se na faixa esperada (FAO, 2009; Silva e Faria, 1995).

A Aeschynomene e o Alysicarpus são capazes de taxas de acúmulo de até $27,9 \mathrm{~kg} \mathrm{ha}^{-1} \mathrm{dia}^{-1}$ e $31,6 \mathrm{~kg}$ $\mathrm{ha}^{-1} \mathrm{dia}^{-1}$, respectivamente. A Aeschynomene não apresenta diferenças na produção entre frequências de corte (Tab. 1), mas tem maior PB e DIVMS nos cortes aos 30 dias. A produção de Alysicarpus é maior nas frequências de corte menores, mas com menor valor nutricional (Tab. 4 e 5). Ambas não se adaptam à sombra. O teor de proteína do Alysicarpus está abaixo de outros relatos (Cook et al., 2005) nos cortes acima de 120 dias; a Aeschynomene está dentro da faixa esperada: Cook et al. (2005) relataram teores de proteína bruta variando de $23 \%$ a $27 \%$ nas folhas e de $9 \%$ a $14 \%$ nas hastes, em amostras dos $15 \mathrm{~cm}$ superiores dessa forrageira. A DIVMS de Alysicarpus é inferior ao relatado (Cook et al., 2005). Não foram encontrados dados para o Aeschynomene.
Assim, confirmou-se a hipótese inicial de que espécies de leguminosas tropicais herbáceas apresentam requisitos específicos de adaptação agronômica que são determinantes do êxito da sua exploração comercial. Além do grau de adaptação local, requisitos específicos de manejo devem também ser considerados para que tal êxito seja alcançado. $\mathrm{O}$ comportamento diferenciado das espécies adaptadas podem ser utilizados , por exemplo, em plantio simultâneo de várias espécies, de forma a assegurar a longevidade da exploração econômica.

\section{CONCLUSÕES}

O Arachis, o Estilosantes, o Calopogonio, a Centrosema, a Pueraria, a Clitoria e o Aeschynomene mostraram melhor desempenho agronômico quando submetidos a frequências de corte de 30 a 90 dias quando cultivados sob pleno sol. Sob condições de sombra, a frequência de corte de 60 dias permitiu melhor desempenho da Centrosema, da Pueraria e do Calopogonio. O Lablab produziu melhor com corte aos 120 dias ao sol, e o Arachis e o Estilosantes produziram melhor com menores frequências de corte à sombra, mantendo o valor nutricional, prestando-se assim para manejo diferido ao sol. O Alysicarpus e a Aeschymomene não toleraram o nível de sombreamento que prevaleceu neste experimento.

\section{AGRADECIMENTOS}

Agradecemos à FAPESP pelo financiamento, ao Prof. Dr. José Leonardo de Moraes Gonçalves pelo apoio e à Dra. Patrícia Perondi Anchão Oliveira pelas sugestões.

\section{REFERÊNCIAS}

ANDRADE, C.M.S.; GARCIA, R.; COUTO, L.; PEREIRA, O.G. Fatores limitantes ao crescimento do capim-tanzânia em um sistema agrossilvipastoril com eucalipto, na região dos cerrados de Minas Gerais. Rev. Bras. Zootec., v.30, p.1178-1185, 2001.

ANDRADE, C.M.S; VALENTIM, J.F.; CARNEIRO, J.; VAZ, F.A. Crescimento de gramíneas e leguminosas forrageiras tropicais sob sombreamento. Pesq. Agropec. Bras., v.39, p.263-270, 2004.

ASSOCIAÇÃO Brasileira de Produtores de Florestas Plantadas (Abraf). Anuário estatístico da ABRAF 2012. Brasília, DF: Abraf, 2012. 
CHIN F.Y. Sustainable use of ground vegetation under mature oil palm and rubber trees for commercial beef production, $1998 . \quad$ Disponível em: <www.fao.org/ag/agp/AGPC/doc/Newpub/Philippine Proceedings/chin.pdf>. Acessado em: 17 jun. 2013.

CHONG, D.T.; TAJUDDIN, I.; SAMAT, A.M.S. et al. Stocking rate effects on sheep and forage productivity under rubber in Malaysia. J. Agric. Sci., v.128, p.339-346, 1997.

CONGDON, R.A.; ADDISON, H. Optimising nutrition for productive and sustainable farm forestry systems - pasture legumes under shade. 2003. Disponível em: <http://eprints.jcu.edu.au/192/1/ Congdon_(2003)_RIRDC_report.pdf.>. Acessado em: 30 mar. 2009

COOK, B.G., PENGELLY, B.C., BROWN, S.D. et al. Tropical Forages: an interactive selection tool. 2005. Disponível em: <http://www.tropicalforages.info>. Acessado em: 28 fev. 2012

FAO. Grassland species profile. [última atualização da página: 2009]. Disponível em: <http://www.fao.org/ ag/AGP/agpc/doc/Gbase/Default.htm>. Acessado em: 12 fev. 2012.

FERREIRA, D.J.; ZANINE, A.M.; SOUTO, S.M.; DIAS, P.F. Capim Tanzânia (Panicum maximum) sob sombreamento e manejo de corte. Arch. Zootec., v.59, p.81-91, 2010.
FONSECA, D.M. DA; MARTUSCELLO, J.A. Plantas forrageiras. Viçosa: Ed. UFV, 2010.

GUENNI, O.; SEITER, S.; FIGUEROA, R. Growth responses of three Brachiaria species to light intensity and nitrogen supply. Trop. Grassl., v.42, p.75-87, 2008.

SILVA, C.M.M.S.; FARIA, C.M.B. Variação estacional de nutrientes e valor nutritivo em plantas forrageiras tropicais. Pesq. Agropec. Bras., v.30, p.413-420, 1995.

SILVA, D.J.; QUEIROZ, A.C. Análise de alimentos (métodos químicos e biológicos). 3.ed. Viçosa: Editora UFV, 2002. 235p.

URBANO, D.; DÁVILA, C.; CASTRO, F. Efecto de la altura y frecuencia de corte sobre tres variedades de maní forrajero (Arachis pintoi) en el estado Mérida. I. Rendimiento y contenido de proteína cruda. Zootec. Trop., v.28, p.449-456, 2010.

VILLARREAL, M.; COCHRAN, R.C.; VILLALOBOS, L. et al. Dry matter yield and crude protein and rumen degradable protein concentrations of three Arachis pintoi ecotypes at different stages of regrowth in the humids tropics. Grass Forage Sci., v.60, p.237-243, 2005 . 